\title{
О РОЛИ ВЫЧИСЛИТЕЛЬНОЙ ТЕХНИКИ В СИСТЕМАХ АВТОМАТИЧЕСКОГО УПРАВЛЕНИЯ
}

\section{Введение}

Первые шаги на пути использования вычислительной техники в системах автоматического управления были сделаны в конце 50-х годов, когда некоторые фирмы, в том числе «Монсанто» и «Тексако», попытались приспособить универсальные цифровые ЭВМ для вычисления, a частично, и для управления рядом быстро меняющихся переменных в системах управления непрерывными химическими процессами.

K этому периоду относится также идея создания, помимо универсальных ӘВМ, и управляющих вычислительных машин (УВМ), машин с более развитыми периферийными устройствами, более скромными процессорами и запоминающими устройствами (3У), но с богатым комплектом устройств сопряжения с управляемым процессом. Одним словом, ученые и инженеры хотели создать компъютер, более подходящий для функций управления, чем универсальная ЭВМ.

К этому времени относятся весьма оптимистические высказывания крупнейших специалистов, например, Н. Вннера, А. И. Берга, Т. Уиллиамса (первым внедрившего ЭВМ в «Монсанто»), о том, что ЭВМ найдут самое широкое применение в системах управления уже в ближайшие годы.

K этому же времени относится и появление первых оригинальных отечественных УВМ (почти одновременно с соответствующими зарубежными), таких как УПП1, ВНИИЭМ, УМ1, «Днепр-1», УМ1-НХ и т. д.

Однако скоро выяснилось, что проблемы, связанные с эффективным использованием ЭВМ в управлении процессами, оказались намного сложнее, чем предполагалось. Ни наука, ни техника, ни технология, ни кадры не были готовы к быстрому решению сложнейшего комплекса этих задач.

Сегодня точно можно выделить два этапа в решении указанных задач. Первый завершился созданием миникомпъютеров, а также разработкой принципов прямого цифрового управления (ПЦУ) и супервизорного управления на базе концепции централизованного управления. На это понадобнлось десять лет.

Реализация же задач второго этапа - речь идет об интегрированных иерархических системах распределенного цифрового управления децентрализованным интеллектом, в которые входят все уровни вычислительной техники, начиная с больших универсальных ӘВМ и кончая микропроцессорными контроллерами, - потребовала двадцати лет работы, и этот этап еще не завершен.

В настоящее время во всем мире работают несколько сотен тысяч автоматизированных систем управления технологическими процессами (АСУ ТП), в которых используются около миллиона различного рода 
вычислительных процессоров. Притом, что примечательно, $75 \%$ из этих систем созданы или введены в действие после 1975 года, т. е. за последние шесть лет.

\section{Централизованные цифровые системы управления процессами}

Роль первых ЭВМ в системах управления производственными процессами сводилась к определению уставок наиболее важных регуляторов аналогового типа для того, чтобы улучшить качественные показатели процесса.

Следующим этапом использования ЭВМ в управлении было ПЦУ. Его централизованный одноуровневой вариант заключался в том, что многочисленные традиционные регуляторы были заменены одним цифровым компъютером (рис. 1). В универсальную ЭВМ такой централизованной системы передаются данные (результаты измерения) от всех датчиков процесса. В ЗУ той же ЭВМ записываются также программы

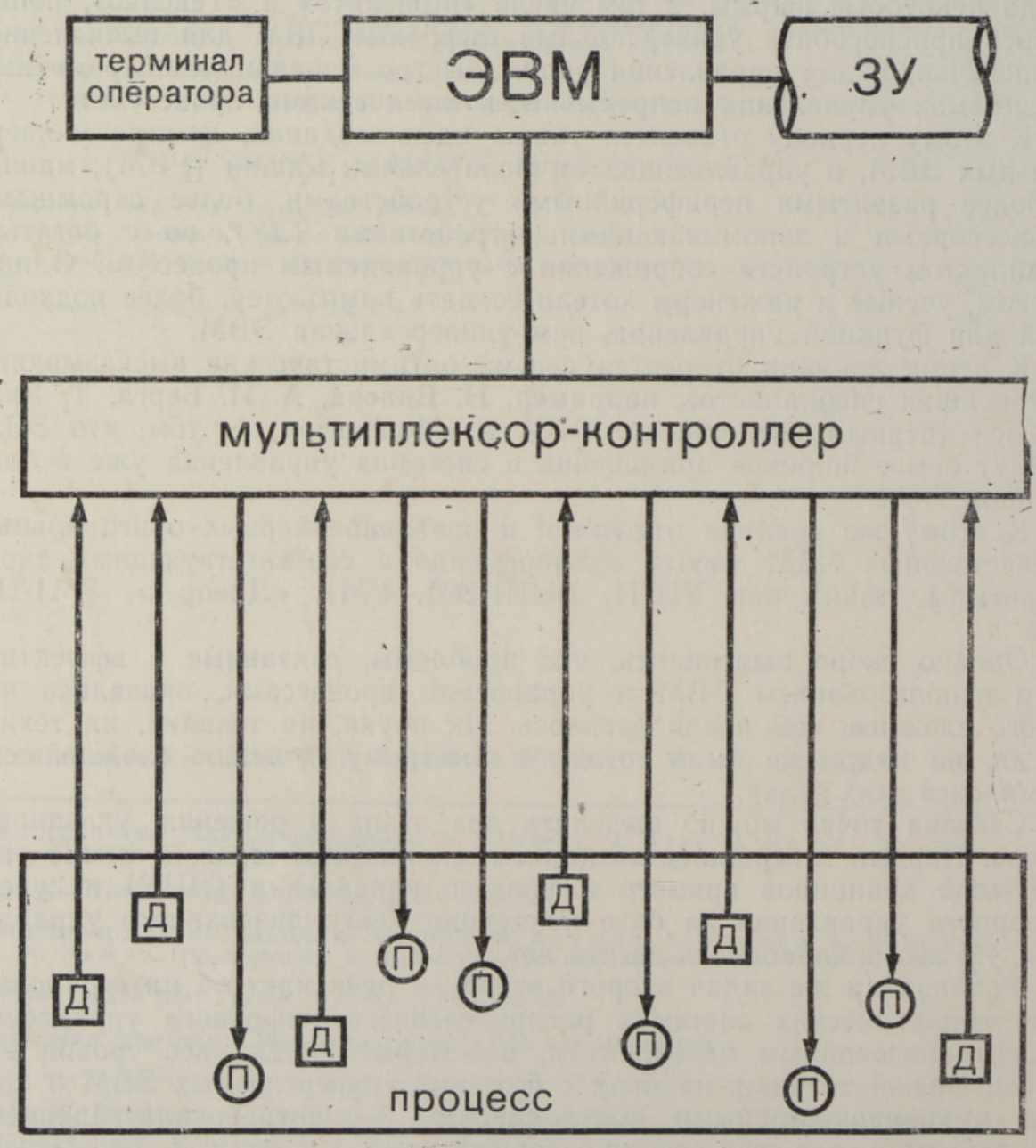

Рис. 1.

Д - датчики, П - приводы исполнительных органов. 
управления, включая и некоторую базу данных. С помощью этих средств, учитывая конкретные результаты измерений, ЭВМ подсчитывает численные значения управляющих воздействий и передает их к исполнительным механизмам. Мультиплексор-контроллер заботится o последовательности подключения к ЭВМ коммуникационных средств - как датчиков, так и приводов исполнительных органов.

Быстродействие такой вычислительной машины уже во многих случаях позволило справиться со всеми контурами обратной связи процесса последовательно, т. е. таким образом, что весь цикл управления оставался в пределах временных ограничений управляемого процесса. Математическое обеспечение этих ЭВМ имело уже солидную базу и было способным реализовать сложные программы управления.

Однако вскоре выяснилось, что ПЦУ, которое реализовалось главным образом по принципу централизованного одноуровневого управления, оказалось перспективным и жизнеспособным лишь в немногих случаях. Почему? В первую очередь из-за ненадежности и дороговизны. Чтобы понять суть этих причин, проанализируем основные функции вычислительной управляющей системы. Этих основных функций три: сбор информации, вычисление значений управляющих воздействий и их реализация, т. е. передача их через устройства сопряжения, системы связи в приводы исполнительных механизмов.

Сбор информащии начинается с преобразования в цифровой код любого переменного управляемого процесса. Далее многое зависит от программного обеспечения, которое преобразует сигнал в необходимую форму, проверяет его попадание в требуемые пределы и передает его в ту часть системы управления, где проводятся вычисления.

Затем, на базе полученной и подготовленной таким образом информации и с помощью соответствующих алгоритмов управления, которые записаны в виде программы в ЭВМ, вычисляются численные значения управляющих воздействий, которые передаются в приводы исполнительных устройств.

Поскольку в природе и в технологических процессах измеряемые сигналы, как правило, существуют в аналоговой форме, а ЭВМ цифровая, то приходится прибегать к двукратному преобразованию: сначала аналого-цифровому, а затем цифро-аналоговому. Используемые при этом преобразователи должны удовлетворять весьма широкому диапазону частот.

В этой связи мультиплексор, находящийся в системе, должен был обслуживать многочисленные переключатели, как правило, аналоговых сигналов к каналу входа ЭВМ. Однако тогдашний уровень электроники не позволял ему справляться с этой задачей удовлетворительно.

Кроме средств обычного сбора информации и произведения вычислений, математическое обеспечение управляющих ЭВМ должно содержать еще средства для реагирования на непредвиденные ситуации, в том числе и аварийные. ЭВМ должна быть способной прерывать процесс управления и вводить экстренные поправки для сохранения устойчивости, а также обеспечивать качественные показатели процесса. Все это, как правило, существенно усложняет программное обеспечение управляющих ЭВМ, вплоть до установления шкал приоритетов непредвиденных событий. Такие системы прерывания часто получаются многоступенчатыми, т. е. одни прерывания могут быть прерваны другими, более высокого приоритета (рис. 2).

Фундаментальным при организации работы ЭВМ, включенной в систему управления, является то обстоятельство, что она должна 


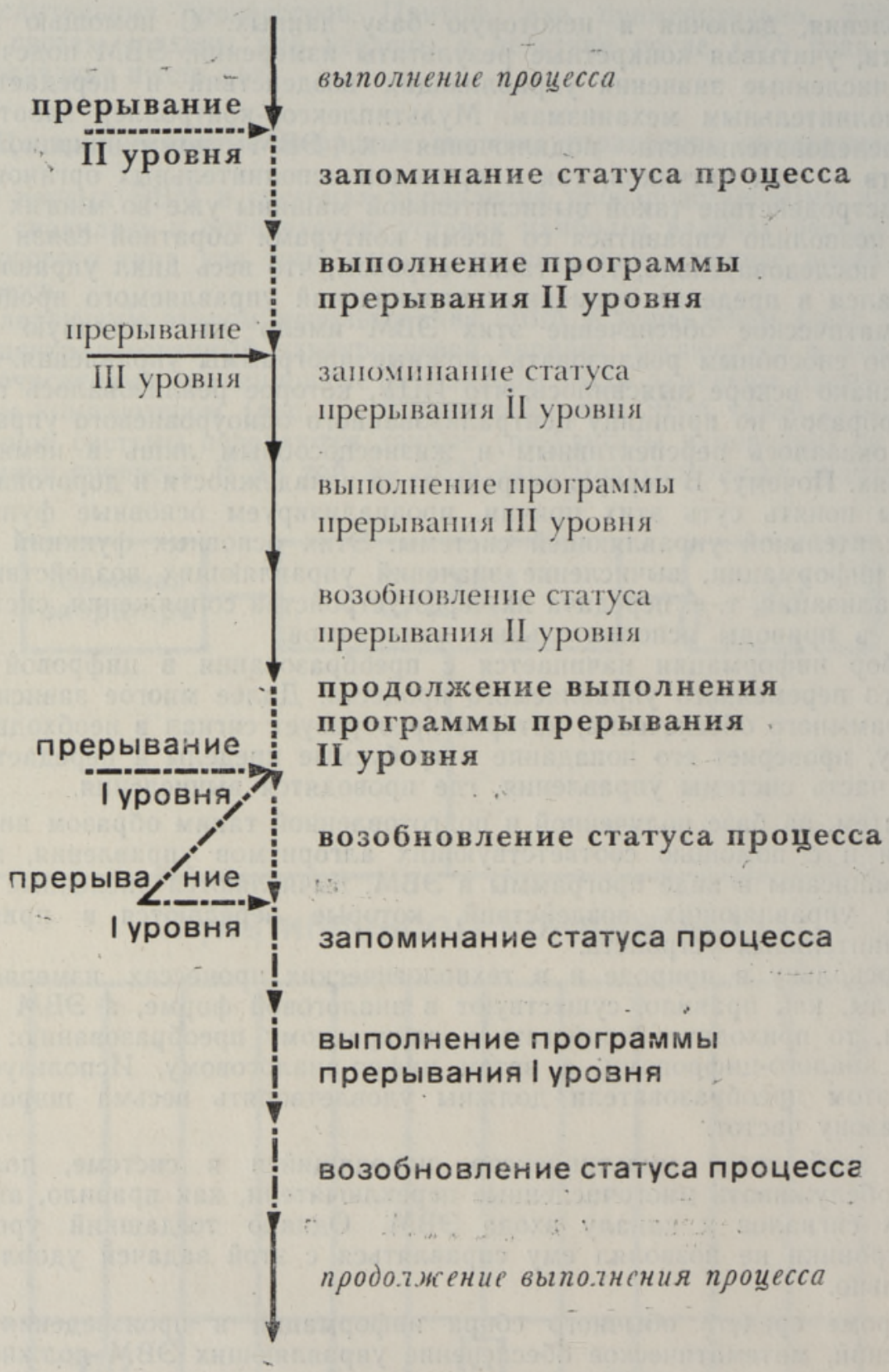

Рис. 2.

работать в т. н. реальном масштабе времени, т. е. должна выполнять свои функции быстрее или же в течение того промежутка времени, который требуется управляемому процессу. Поэтому такая ӘВМ оснащена часами, проверяющими ее статус много раз в секунду.

Кроме того, компъютер должен выполнять мультипрограммную работу с описанными шкалами приоритетов прерывания вычислительного процесса. Работа УВМ, в отличие от других ЭВМ, главным образөм диктуется внешними событиями, которым должны соответство- 
вать не только аппаратурная часть, но и операционные системы и управляющие программы. Поэтому не удивительно, что отсутствие необходимого математического обеспечения стало основной причиной задержки реализации сложных АСУ ТП, что стоимость этого «мягкого» продукта, управляющих программ, превысила стонмость современнейшей аппаратуры.

Первоначальный оптимизм относительно цифрового управления заметно охладили и другие обстоятельства.

Такая система управления, в которой центральной частью служила большая универсальная вычислительная машина, оказалась весьма дорогостоящей и доступной по этой причине не каждому заводу и не каждому процессу.

С другой стороны, поскольку весь дорогой и многотоннажный производственный процесс отныне стал полностью зависеть от ЭВМ, а эта последняя не была достаточно надежной, то такие управляющие системы стали либо дублировать, либо подстраховывать при помощи многочисленных систем аналоговых регуляторов. В обоих случаях стоимость систем управления поднялась до стоимости самого технологического процесса, а надежность, в общем, улучшилась только незначительно.

К числу сдерживающих широкое распространение ПЦУ причин следует причислить и те, которые связаны с программным обеспечением и которые выявлялись только по мере того, как стало исчезать наивное представление о том, что программирование - это несложное занятие.

Так, например, в период централизованного ПЦУ математическое обеспечение по технической диагностике фактически отсутствовало. А попробуйте представить себе работу отмеченных здесь подстрахованных сложных управляющих систем без этих средств.

Далее. Система математического обеспечения такого централизованного компъютера управления, которая должна управлять центральным процессором, обслуживая многочисленные датчики и приводы, управлять динамическим распределением 3У, обеспечивать своевременную бесконфликтную коммутацию входов-выходов, регулировать все хозяйство, связанное с базой данных, оказалась уже настолько сложной, что даже если ее в отдельных случаях и удавалось разработать, то она фатально ухудшала основные качества аппаратурной части системы управления, например, время реакции. Вместо $4-$ 10 мкс, которые под силу «хардвару», управляющая программа увеличила это время до $200-300$ мкс, т. е. от двадцати до восьмидесяти раз.

Здесь уместно напомнить: для того, чтобы человечество получило первую, полностью отлаженную, апробированную и надежную универсальную систему программирования (ФОРТРАН), потребовалось ровно двадцать лет. А для исследования, разработки, тестирования ПЭАРЛ одного из наиболее популярных в Западной Европе языков программирования в реальном времени - к началу этого года было израсходовано более 30 миллионов долларов, а работы по его отладке все еще продолжаются.

Важным фактором использования вычислительной техники в системах автоматического управления было появление в середине 60 -х годов управляющего миникомпъютера. Это позволило перейти от одноуровневого управления к иерархическим системам, где исполнение отдельных управляющих воздействий, а также сбор и некоторое агрегирование первичной информации были распределены между несколькими 


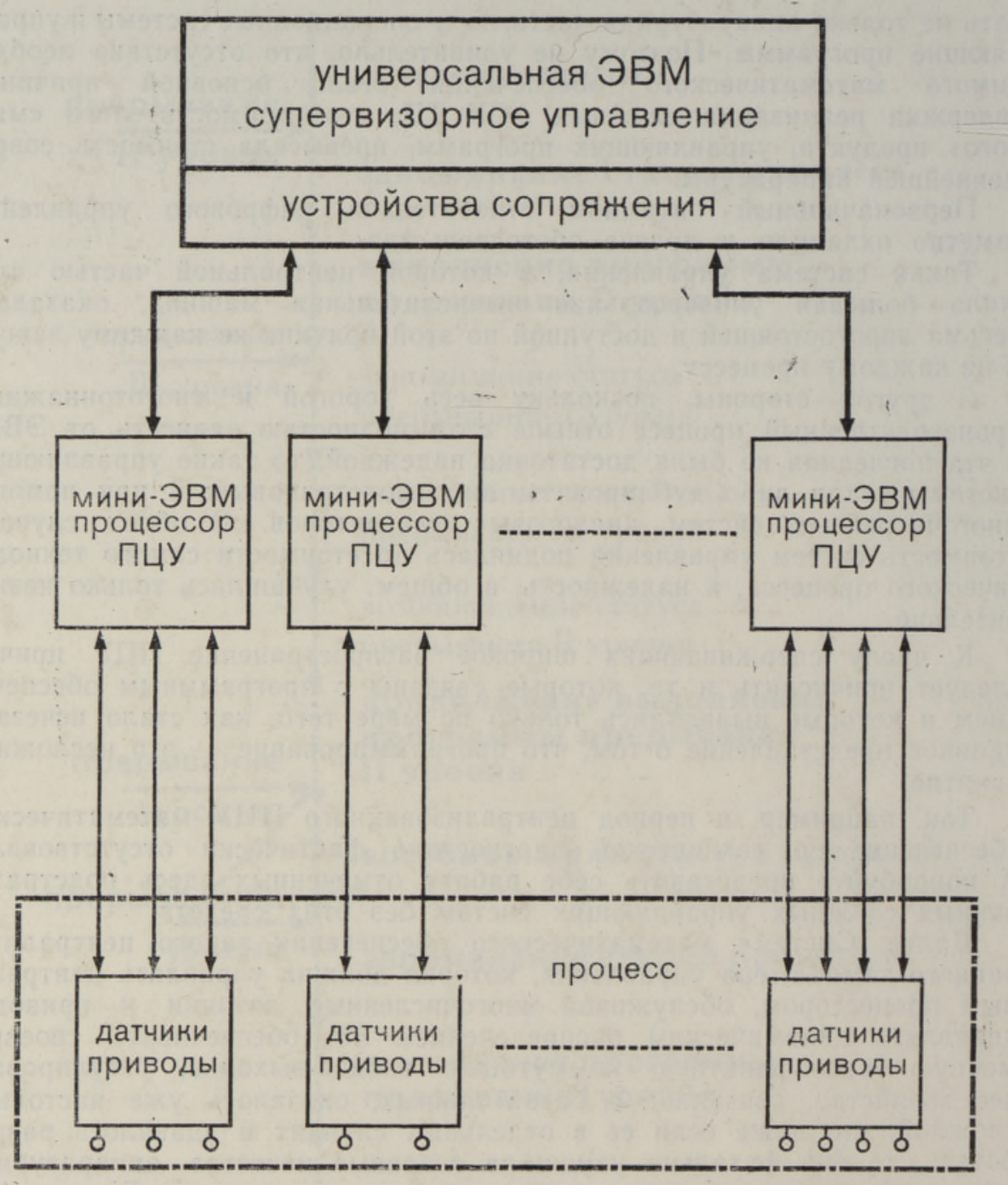

Pис. 3.

мини-ЭВМ. Само же управление осталось централизованным и обеспечивалось центральной ЭВМ универсального типа (рис. 3). В такой системе управления мини-ЭВМ, находящиеся на первом уровне управления, выполняют функции процессоров ПЦУ. Центральная же ӘВМ выполняет т. н. супервизорные функции или функции каскадного регулирования. Такая архитектура системы управления оказалась более гибкой и в ряде случаев вполне жизнеспособной, однако широкого самостоятельного распространения она все же не получила. Почему? Можно назвать три решающие причины.

Во-первых, наличие большого центрального дорогостоящего компъютера заставляло заботиться о его эффективном использовании. Другими словами, функции управления были подчинены в ущерб эксплуатации процесса интересам эксплуатации ЭВМ. Да и функции еще какого управления - централизованного и сосредоточенного как в функциональном, так и пространственном и структурном смысле, 
которое из-за негибкости, больших постоянных времен обратной связи, ненадежности, нецелесообразных требований к архитектуре программного обеспечения и еще из-за ряда других причин начало изживать себя.

Во-вторых, в супервизорной системе комплекс устройств сопряжения вместе с линиями коммуникации (кабелями) получился неоправданно материалоемким и дорогим.

В-третьих, в результате быстрого технического прогресса в микроэлектронике появились дешевые микропроцессоры на больших интегральных схемах, которые позволили перейти к реализации гораздо более прогрессивных концепций и плодотворных идей в управлении большими системами.

\section{Распределенные системы управления}

В начале 70-х годов произошел очередной качественный скачок в развитии элементной базы вычислительной техники. Появились схемы со средней и большой степенью интеграции, новые ЗУ на полупроводниках, целые микропроцессоры на одном кристалле.

В результате этого мини-ЭВМ перестали быть минимашинами по мощности и вычислительной способности (они остались мини-ЭВМ только по габаритам). Помимо миникомпъютеров, появились микропроцессоры и микро-ЭВМ. Эти технические новшества внесли кардинальные коррективы в принципы, методы и структуру управления процессами и системами. Они привели прежде всего к различным распределенным и децентрализованным системам, в которых по сравнению с вышеупомянутыми сосредоточенными системами гораздо качественнее и эффективнее реализуются такие фундаментальные параметры управления, как надежность, устойчивость, гибкость и быстродействие обратной связи, задачи оптимизации.

Переход от централизованных к распределенным и комбинированным системам и структурам позволил отразить иерархичность самой структуры управления, позволил распределить между модулями системы не только функции управления, но и интеллект. С этим переходом связаны также распределение обработки информации, перемещение средств обработки информации к их источникам, интерактивность различных модулей одного и того же уровня системы управления, а также появление человека-оператора и высокая степень параллелизма в вычислительном процессе.

Такая децентрализованная система с распределенными функциями управления, использующая четыре различных уровня вычислительной техники, представлена на рис. 4.

Данный пример построен на базе модели управления заводом, где на самом низком уровне многочисленные микропроцессорные устройства осуществляют сбор первичной информации, а также контроль над отдельными переменными процессами. Такие контроллеры, которые работают, как правило, без вмешательства человека, группами подключаются к микро-ЭВМ следующего, в нашем примере, цехового уровня.

Микрокомпъютеры цехового уровня выполняют ряд ответственных задач. Они обрабатывают первичную информацию, поступающую непосредственно с датчиков процесса, общаются с помощью т. н. интерактивных переменных с другими модулями того же уровня, оперативно согласовывая локальные функции управления; в новейших системах на этом уровне все чаще встречаются диалоговые подсистемы, т. е. интеракции с человеком-оператором, и локальные сети микро-ЭВМ, которые часто выполняют функции нижнего звена подсистемы ПЦУ, 


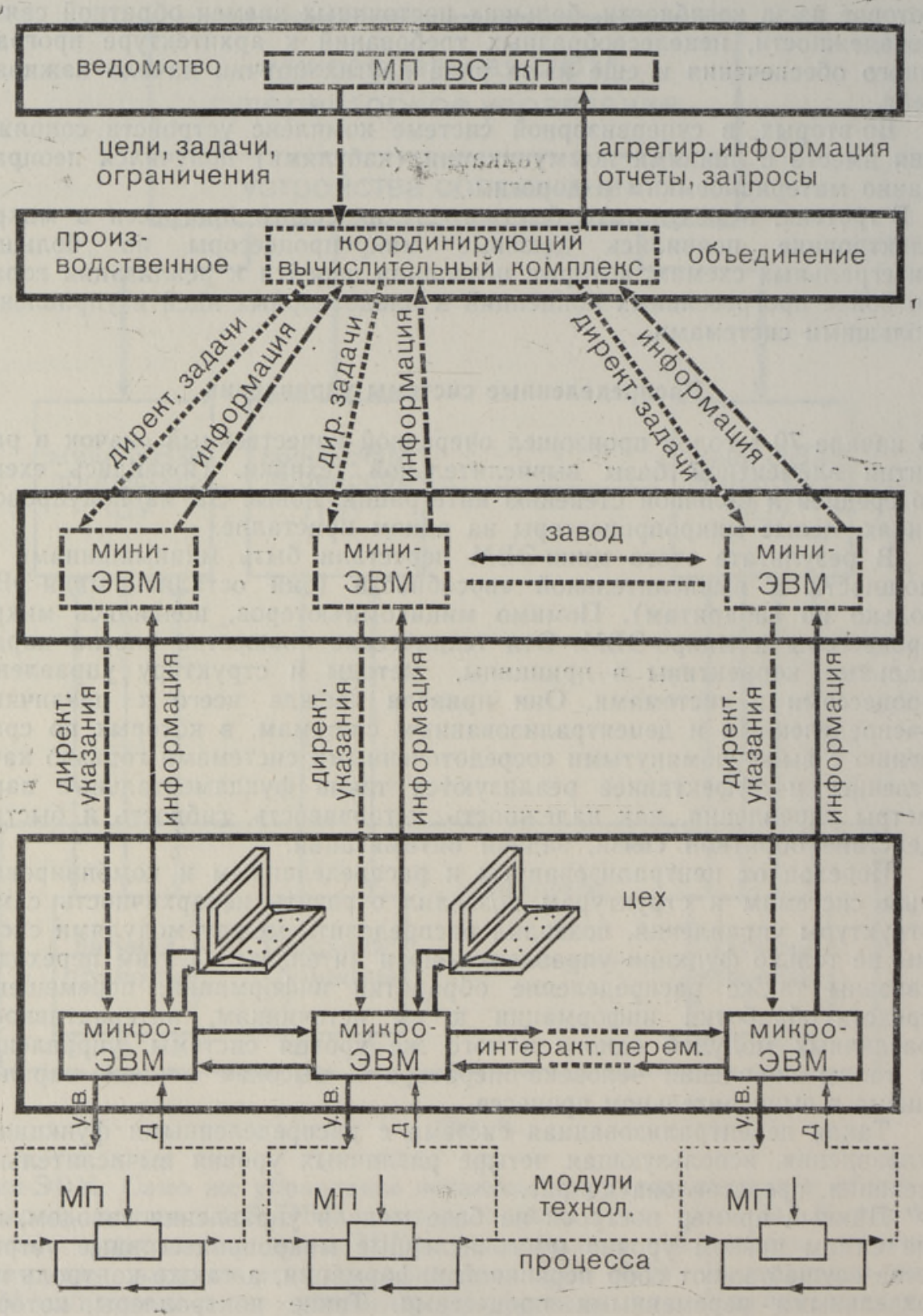

Рис. 4.

УВ - управляющее воздействие, Д - данные, МП - микропроцессор.

передавая обработанную первичную информацию в ЭВМ следующего уровня и реализуя поступающие оттуда директивы. Разумеется, не всегда все перечисленные здесь функции должны обязательно быть возложены на компъютеры цехового уровня.

На следующем - заводском - уровне без современных миникомпъютеров не обойтись. До сих пор, как известно, в отечественных 
системах на этом уровне широко использовались универсальные ЭВМ, но теперь целесообразно от них отказаться. В этой связи процитируем академика А. П. Александрова, который весной 1979 года на пленуме Координационного комитета АН СССР по вычислительной технике сказал: «Универсальные машины надо применять там, где они действительно необходимы, а в остальных случаях целесообразнее применять микропроцессорную технику, которая намного дешевле, экономнее универсальных машин, и в ней нет лишней избыточности».

Это на самом деле соответствует действительности. В предыдущем разделе было показано, что для обеспечения надежности в централизованных системах с универсальными ЭВМ приходилось дублировать всю систему управления, а в распределенных системах с микропроцессорной техникой можно ограничиться дублированием только нанболее ответственных и наиболее критических модулей нижних звеньев. Это позволяет минимизировать редундантность, или избыточность, и иметь все вытекающие отсюда положительные последствия.

Таким образом, на уровне завода реализуются цели управления, поставленные координируюшей вычислительной машиной, находящейся, например, на уровне производственного объединения, с учетом местных ограничений и условий.

Мини-ЭВМ на заводском уровне, разумеется, могут быть включены в общую структуру управления и иными, чем показано на рис. 4, способами. В нашем примере имеется в виду, что превалирует необходимость реализации локальных подсистем ПЦУ. Но конкретные ситуации могут потребовать совсем иных решений.

Здесь уместно напомнить, кстати, неоднократные высказывания академика Г. И. Марчука о том, что при проектировании какой-либо системы на уровне предприятия или объединения мы, как правило, считаем, что АСУ - это одна линия, АСУ ТП - другая, каждая со своей вычислительной техникой. Наличие же микрокомпъютерной техники позволяет объединить эти две линии, интегрировать цели административно-экономические с целями техническими и технологическими.

Это, конечно, серьезнейшая задача прежде всего для системных инженеров, и системных программистов, а также для ученых. Например, в настоящее время не имеют универсального решения такие задачи, как автоматическая обработка данных с произвольной структурой, управление крупными банками данных без потери быстродействия и т. п. Одним словом, здесь много проблем, которыми мы должны заняться в ближайшие годы.

Что касается самых верхних уровней распределенной системы, то здесь наиболее перспективны универсальные ЭВМ коллективного пользования, которые получают агрегированную, сводную информацию от более крупных единиц управления, а на более низкие уровни передают, как правило, комплексные, укрупненные задания с более общими ограничениями.

Так, например, на уровне объединення в качестве координирующей ЭВМ может выступать либо самостоятельная машина, либо интеллигентный терминал (или терминалы), либо сателлитная машина крупной многопроцессорной вычислительной системы коллективного пользования, которая сама находится на ведомственном уровне.

Уже на более низких уровнях, не говоря уже о высоких, возникают новые сложные проблемы в связи со структурами, топологией, совместимостью и т. п., которые уже относятся к разделу вычислительных сетей. 


\section{Вычислительная техника и системы автоматизации проектирования систем управления (САПР СУ)}

Среди многих причин очень скромного использования вычислительной техники в системах автоматического управления вопросы проектирования указанных систем занимают особое место. Поэтому коротко остановимся на них.

До недавнего времени ПИД-регулятор был основным управляющим звеном в самых различных системах управления. Это означает, что современная теория управления еще не привлекла серьезного внимания инженеров в области управления технологическими процессами и что между теорией и инженерной практикой существует явный разрыв. Широкое распространение дешевых микро-ЭВМ решительно изменило ситуацию, создало условия для замены полувековых методов и алгоритмов более развитыми и эффективными. Появление микро-ЭВМ пробудило значительный интерес к многомерным и адаптивным системам автоматического управления, поскольку взаимосвязанность и неопределенность являются существенными характеристиками современных многоступенчатых технологических процессов.

Системы автоматического управления, содержащие вычислительную технику, как мы видели, относятся к сложным системам. Поэтому их ручное проектирование не может, как правило, увенчаться успехом. Необходимо перейти на машинное проектирование, т. е. использовать для этого саму вычислительную технику. Это, однако, чрезвычайно сложно, хотя бы потому, что проектирование до сих пор относится, несмотря на наличие многих норм и стандартов, к проблемам чисто эмпирическим, решаемым на основании инженерной интуиции и опыта. Вместе с тем уровень современной вычислительной техники требует сейчас строгой формализации процесса проектирования.

Помимо явных методологических трудностей, успешному решению машинного проектирования мешает еще ряд совершенно принципиальных объективных причин. Среди них недопонимание практических проблем управления со стороны разработчиков систем управления процессами. Так, например, разработчики страстно и безуспешно стараются найти какой-нибудь единый критерий подсчета оптимальных режимов пронзводственного процесса, забывая о том, что на самом деле важнейшей проблемой является живучесть процесса, его непрерывное функционирование, его надежность, т. е. что единственно верным является многокритериальный подход.

Далее. Проектирование технологического процесса до сих пор, как правило, осуществлялось отдельно от проектирования соответствующей системы управления ЭВМ. Такое положение, в свою очередь, было обусловлено объективными причинами: отсутствием достаточно подходящих аппаратурных (в том числе и УВМ) и программных средств, как для управления, так и для проектирования, и отсутствием специалистов требуемого профиля. Только сейчас пришли на работу первые инженеры, получившие высшее образование по управляющим вычислительным машинам и системам, а проектировщиков систем управления процессами до сих пор нет.

Все это, в конце концов, не так уж удивительно. Ведь при проектировании технологического процесса вместе с системой его управления появляется клубок требующих решения проблем - начиная от исследования объекта (рис. 5), его моделирования, преобразования этих моделей в комплекс различных программ, структурирования архитектуры системы управления вместе с аппаратурной частью, включая ӘВМ 


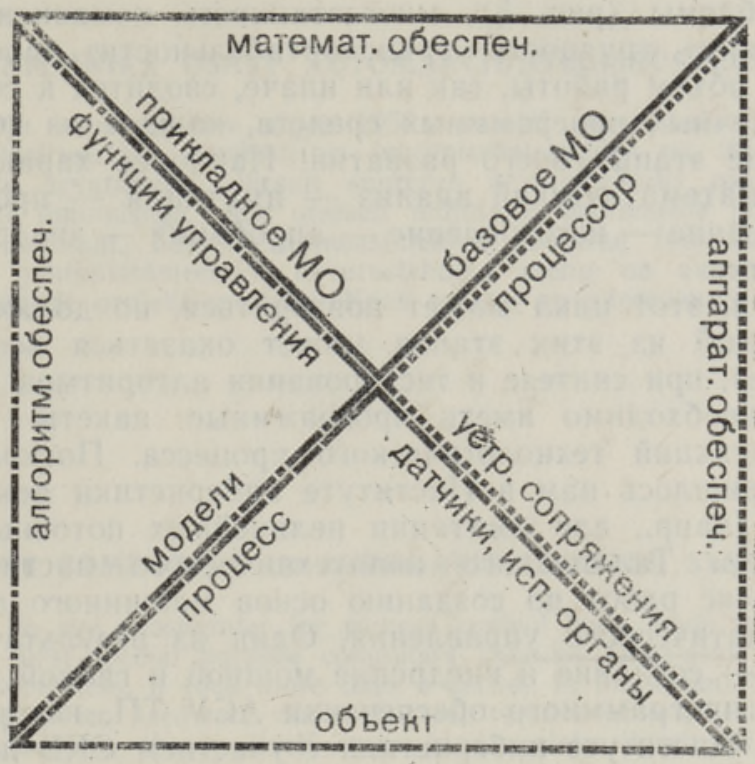

Рис. 5.

всех необходимых категорий, и кончая успешным завершением этого процесса, т. е. кончая реализацией всех алгоритмов через аппаратурные части так, чтобы обеспечить надежное функционирование объекта как в стационарном режиме, во время переходных процессов, так и в аварийных ситуациях.

Рассмотрим показанную диаграмму (введена Р. Тавастом, Ин-т кибернетики АН ЭССР) подробнее.

Первый комплекс вопросов, как уже сказано, заключается в формализации объекта и процесса с точки зрения проектирования. Для этого необходимо иметь все модели объекта и процесса, цели и функции управления в виде дискретных алгоритмов, преобразуемых в дальнейшем в модули математического обеспечения в качестве прикладных программ.

Машинному проектированию естественно присуща значительная интерактивность, т. е. диалог человека с машиной, многократная оценка промежуточных результатов, проигрывание альтернативных вариантов, экономический анализ, вплоть до подготовки проектной документации.

В области автоматизации проектирования больше всего сделано пока в машиностроении и в «регулирующей автоматике». В последние годы отдано много сил автоматизации проектирования прикладного программного обеспечения. Для этого необходимо было создать и разработать сложные инструментальные средства программирования. Хотя и эти вопросы далеки от завершения, нельзя не сослаться на хорошие результаты Института кибернетики АН ЭССР, которые заключаются в практической реализации двух различных инструментальных систем МЕМО-ИСП и ПРИЗ, генерируюших прикладные языки и программы такого рода. Әти системы всячески апробированы и имеют свою клиентуру как в нашей стране, так и в других социалистических странах.

Проектирование систем управления является многофакторным процессом, где одновременно должны быть учтены все четыре основные 
стороны проблемы (рис. 5), многоэтапность компонентов, входящих в «треугольники», трудность «транспортабельности» проектов.

Основной объем работы, так или иначе, сводится к созданию и разработке различных программных средств, каждое из которых должно пройти многие этапы своего развития. Нанболее характерные из них следующие: математический анализ - имитация - проектирование документирование - изготовление - апробация - внедрение - оценка эффективности.

Разумеется, этот цикл может повторяться, но должен быть сходящимся. Каждый из этих этапов может оказаться весьма сложным. Так, например, при синтезе и тестировании алгоритмов супервизорного управления необходимо иметь программные пакеты для имитации некоторых функций технологического процесса. Поскольку таких не оказалось, пришлось нам в Институте кибернетики некоторые из них разработать (напр., для имитации нелинейных поточных сетей). Там же с участием Таллинского политехнического института проведен целый комплекс работ по созданию основ машинного проектирования систем автоматического управления. Один из результатов этой большой работы - создание и внедрение мощной и гибкой инструментальной системы программного обеспечения АСУ ТП, которая называется МЕДИУС. В Институте кибернетики с участием СКБ по вычислительной технике разработана еще диалоговая система машинного проектирования многомерных микропроцессорных регуляторов, нашедшая применение при проектировании микропроцессорной системы автоматического управления зерносушилкой, лазерным спектрометром, а также при обучении студентов.

Разумеется, эти несколько примеров разработки отдельных элементов всеобъемлющей САПР СУ можно дополнить многими другими. Однако если принять во внимание все необходимое множество вопросов, которое должно быть решено в целом для создания САПР СУ, то, надо признать, в настоящее время «белых пятен» на этом множестве больше, чем изученных и освоенных областей.

В заключение хотелось бы просто повторить истину, что за последние два десятилетия вычислительная техника сыграла существенную роль в развитии автоматического управления, причем ее роль все время возрастает. Она повлияла не только на технику реализации управления, но и на методы исследования и создания систем управления. Кроме того, она решительно изменила фундаментальные концепции автоматического управления и управления в целом, открыла новые возможности, в частности, при управлении сложными системами и сверхбыстропротекающими процессами.

Интенсивное развитие вычислительных управляющих систем, переход от сосредоточенных и централизованных к рассредоточенным и децентрализованным системам, многозначность такого рассредоточения и децентрализации, а также богатейшие технические возможности реализации преимуществ тех или иных принципов в ближайшем будущем приведут, скорее всего, к комбинированному использованию всех интеллектуальных и технических средств.

Разумное комбинированное использование теорин и техники управления, имеющихся уже сегодня в нашем распоряжении, должно дать изящные решения многих проблем уже в скором будушем.

таллинский политехнический институт

Поступила в редакцию $5 / \mathrm{V} 1981$ 


\section{B. TAMM}

\section{ARVUTUSTEHNIKA OSAST AUTOMAATJUHTIMISSUSTEEMIDES}

Arvutustehnika mõju automaatikale algas 1950. aastatel universaalarvutite mõningase kasutamisega tsentraliseeritud numbrilistes juhtimissüsteemides nii infotöötlemisel kuı ka juhtimissuuruste arvutamisel. Järsult suurenes arvutustehnika osa 1970. aastatel seoses mikroarvutite ilmumisega, mis otseselt mõjutasid protsesside juhtimise teooriat, kontseptsioone ja tehnikat. Levisid mitmeastmelised jaotatud funktsioonide ja intellektiga paindlikud juhtimissüsteemid. Arvutustehnika areng on avaldanud otsustavat möju juhtimissüsteemide projekteerimisele. Koos sellega on suurenenud programmvarus. tuse osatähtsus.

\section{B. TAMM}

\section{ROLE OF COMPUTERS IN AUTOMATIC CONTROL SYSTEMS}

The first attempts to use a computer for process control were made in the late fifties. At the same time first special purpose computers (besides large-scale ones) appeared in the market. Nevertheless, it took more than a decade to implement computer controi effectively since computer hardivare and software, communication techniques and the corresponding engineering theory itself were inadequately developed to solve such a complicated problem.

The first computer control systems were based on centralized direct digital control. The availability of minicomputers in the late sixties provided for handling each level of the control hierarchy more autonomically. Interactive and displaying technique supported by the corresponding software opened the door for distributed computer control and data processing including real-time control systems.

The seventies featured a great step forward in regard to integrated circuit electronics and microprocessors.

Some principal concepts of computer aided design are discussed in the concluaing part of the paper. 